\title{
LUT
}

University

\section{Topology Optimization of Micro-robotic Appendages Combining Piezoelectric, Polymer, and Silicon Beams}

\author{
Milojevic Andrija, Krokhmal Vladimir, Wu Bingbing, Oldham Kenn
}

This is a Final draft

version of a publication

published by IEEE

in 2019 International Conference on Manipulation, Automation and Robotics at Small Scales (MARSS)

DOI: $10.1109 /$ MARSS.2019.8860976

Copyright of the original publication: (C) 2019 IEEE

Please cite the publication as follows:

A. Milojević, V. Krokhmal, B. Wu and K. Oldham, "Topology Optimization of Micro-robotic Appendages Combining Piezoelectric, Polymer, and Silicon Beams," 2019 International Conference on Manipulation, Automation and Robotics at Small Scales (MARSS), Helsinki, Finland, 2019, pp. 1-6, doi: 10.1109/MARSS.2019.8860976.

(C) 2019 IEEE. Personal use of this material is permitted. Permission from IEEE must be obtained for all other uses, in any current or future media, including reprinting/republishing this material for advertising or promotional purposes, creating new collective works, for resale or redistribution to servers or lists, or reuse of any copyrighted component of this work in other works.

This is a parallel published version of an original publication. This version can differ from the original published article. 


\title{
Topology Optimization of Micro-robotic Appendages Combining Piezoelectric, Polymer, and Silicon Beams
}

\author{
Andrija Milojević, Vladimir Krokhmal, Bingbing Wu, and Kenn Oldham
}

\begin{abstract}
A topology optimization approach is proposed for design of micro-robotic appendages containing active, passive compliant, and rigid beam elements. These elements represent materials and structures - thin-film piezoelectric actuators, parylene-C polymer microstructures, and silicon beams - that have recently been co-fabricated in prototype millimeter scale walking robots. Topology optimization is performed using design synthesis methods that prune an initial network of beams while converting passive compliant beams to active or rigid links using discrete variables. Sample optimization function is introduced for maximizing displacement in two directions subject to certain load bearing constraints. Controllability of multi-direction motion is also optimized through activation of separate piezoelectric elements. Sample design results generated by the proposed algorithm are presented.
\end{abstract}

\section{INTRODUCTION}

Thin-film piezoelectric materials offer several potential benefits for actuation in autonomous micro-robots [1]. Thin-film lead-zirconate-titanate (PZT), for example, has one of the highest work densities per unit area among micro-scale transducers [2]. With sub-micron to a few microns of layer thickness, large electric fields may be generated at modest voltages, simplifying circuit and power source design. As a small capacitive load [3, 4], piezoelectric power consumption and efficiency is generally an advantage relative to other high-force transduction mechanisms suggested for autonomous micro-robots, such as shape memory alloy or electrothermal techniques $[5,6]$.

Disadvantages of thin-film piezoelectric actuators include relatively complex fabrication processes, fragile piezoelectric elements, and modest intrinsic stroke length. Recently, appendages based on combinations of thin-film PZT, high-aspect ratio parylene-C beams, and rigid silicon links (Fig. 1) have been demonstrated that greatly improve robot leg resilience and leverage PZT displacement into a combination of larger-angle inplane or out-of-plane motions [7, 8].

However, this architecture raises questions of how to best design robot appendages to make use of the candidate materials and structures. Although previous iterations of these appendages are designed to resemble appendages of biological organisms, typically insects (Fig. 1a), material properties and transducer range of motion are still not closely matched to those typical among animals.

A. Milojević is with the Department of Mechanical Engineering, Lappeenranta University of Technology, Lappeenratna, Finland (phone: +358465389189; e-mail: Andrija.Milojevic@lut.fi).

V. Krokhmal and B. Wu are with the Department of Mechanical Engineering, University of Michigan Ann Arbor, MI USA.

$\mathrm{K}$. Oldham is with the Department of Mechanical Engineering, University of Michigan Ann Arbor, MI USA (e-mail: oldham@umich.edu).
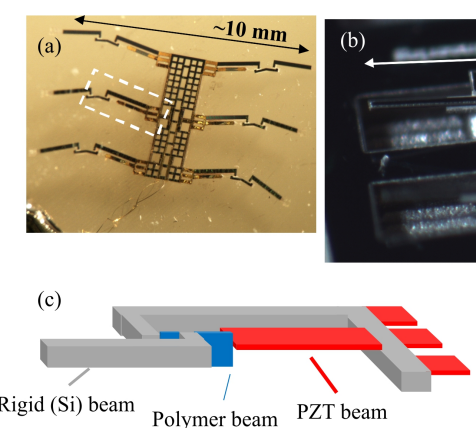

(c)

Rigid (Si) beam Polymer beam PZT beam

Figure 1. (a) Micromachined robot prototype based on thin-film piezoelectric actuators with sample appendage mechanism marked; (b) appendage test structure composed of PZT; (c) schematic arrangement of PZT, polymer, and silicon structures in sample appendage; (d) abstracted structure arrangement in topology optimization framework.

This paper explores possible appendage geometries when topology optimization techniques for compliant mechanisms are applied to the proposed material set of thin-film PZT active elements, compliant polymer beams, and rigid silicon links. The selection of candidate elements is based on those previously fabricated in [7], and as convenient for microstructure release from silicon wafers based on past author experience. Explicitly combining both active piezoelectric and passive polymer element behaviors in design optimization, proposed appendages may be capable of realizing, large, controlled deformations in multiple directions. Thus, compliant mechanisms [9-14] are utilized here for micro-robot appendage development.

The design of compliant mechanisms has been well-studied for the purpose of optimizing elastic structures to accomplish specific tasks. Compliant mechanisms represent monolithic structures that utilize elastic deformation of their segments (elements) to realize force and motion transmission [12-15]. There are many advantages of compliant mechanisms over traditional rigid-body mechanisms, such as reduced complexity, ease of manufacture, avoidance of assembly tasks, zero backlash, friction-free motion, and good scalability. Compliant mechanisms are especial usefully as functional elements in micro-domain applications $[7,9,16]$. The synthesis of compliant mechanisms has usually been done via topology optimization approaches that are well studied [12-19]. For micro-robot design, novelty lies in discrete topology optimization of multiple materials including simultaneous topology optimization of beam like compliant polymer (parylene-C) elements, rigid silicon ( $\mathrm{Si}$ ) elements and active PZT elements/actuators. Optimization includes finding the best arrangements/distribution (number, location, position, orientation) and dimensions (width, thickness, length) of PZT, Si and parylene-C elements so that a micro-robot can realize desired motions of the end-effector. This represents a 
complex optimization problem that cannot be directly solved with existing topology optimization methods.

Multiple-material topology optimization has been done in $[10,17]$ but not including simultaneous optimization of actuator placement (active elements). Moreover, [10, 17] only developed a compliant mechanism for use with an external input. In [18], an effective method for simultaneous topology optimization and actuators placement was introduced, but just for some general actuator type and a single material. In [20] block-based method for topology optimization of passive/active elements is introduced, but with resulting structures that have limited performance due to limitations of the method. Moreover, the problem of control of an end-effector in multiple directions was not addressed. Method presented in [21] focus only on synthesis of microgrippers by utilizing continuum synthesis approach, which is not applicable for discrete optimization problems.

This paper introduces optimization techniques with multiple objectives for developing micro-robot appendages that can control their end-effector in multiple directions when different actuators (PZT elements) are active. The proposed method incorporates simultaneous topology optimization of multiple passive materials (parylene-C and Si elements) and active elements (PZT actuators), optimization of elements widths (PZT, Si, and parylene-C elements), length of elements (by node wandering optimization $[9,15]$ ), and elimination of intersecting overlapping elements $[13,18]$ over large deformations, all in one synthesis process. A method for solving such complex optimization problem has not been developed before in the field of compliant mechanism topology optimization.

\section{METHODS}

In this section, we describe the material elements, design synthesis methodology, and problem specification and solution steps used to generate proposed micro-robotic appendages.

\section{A. Synthesis Methodology}

The synthesis methodology for the current micro-robot application is mainly derived from principles of compliant mechanism design. These mechanisms are usually designed by a continuum synthesis approach [9-19] that includes topology optimization of mechanism and dimensional synthesis (width, thickness, and length) of individual mechanism segments.

Here, the goal is to optimally design a micro-robot structure that can control its appendage end-effector in different directions (in space / in-plane and out-off-plane) when different PZT actuators are active while realizing large displacement of the end-effector. From a topological optimization perspective, the micro-robot structure is formed from:

(1) active elements/actuators, as thin-film PZT beams with metal electrodes that contract and bend;

(2) rigid elements, formed where substrate silicon is retained;

(3) passive elastic/compliant elements, formed from parylene-C. These are formed from combined surface deposition and trench refill, forming beams with either T- or L-shaped cross-sections.
Practically speaking, these elements correspond to the types of elements that have been co-fabricated with thin-film PZT actuators in [7]. Nominally, rigid elements are intended to perform substantial weight bearing with limited compliant structures expected to amplify small piezoelectric input strokes, without exposing piezoelectric elements to excessive forces from robot weight-bearing. However, only a small design space has been explored for mechanisms based on these materials.

Design synthesis in this context includes finding the optimal topology i.e. arrangements/distribution (number, location, position, orientation) and dimensions (width, thickness, length) of PZT, Si and Parylene elements (T or L type) so that a micro-robot can realize desired motions of the end-effector. In topology optimization the designer needs only to define the space in which structure should fit and desired input/output parameters. Then, through optimization, micro-robot appendage structure is automatically generated.

The topology optimization process for the micro-robot appendage includes the following steps:

- $\quad$ setting the problem specifications - defining the desired input parameters,

- parameterization - translating the problem specification into terms/variables for optimization,

- optimization - applying search algorithm for finding the optimal solution of a given formulation.

Based on this, computer-coded algorithm is developed for the optimal synthesis of proposed appendages. The steps of the synthesis process are described in the following sections.

\section{B. Problem Specification}

In the first step of synthesis process, problem specifications are defined (Fig. 2a). This includes defining:

- $\quad$ size and shape of the design domain (space in which micro-robot appendage structure should fit); here a rectilinear design domain is adopted,

- location of the fixed supports of the micro-robot appendage structure,

- location of the end-effector for which motion is controlled, and desired directions of end-effector motion (in-plane/out-off-plane); here both in-plane and out-off-plane directions are selected,

- external loads that act on the micro-robot appendage structure; external loads are applied at the end-effector point, both in in-plane and out-off-plane direction,

- value of input voltage for activating the PZT elements,

- net material properties (Young modulus) of PZT laminate, $\mathrm{Si}$ and parylene-C elements,

- desirable range of width of PZT, Si and parylene-C elements, dictated by constraints on fabrication,

- number of PZT and Si elements that will be used in the micro-robot structure, 
- $\quad$ type of parylene-C elements ( $\mathrm{T}$ or L shaped elements); here both types are used,

The parameters that are used for the synthesis of the microrobot appendages are given in Table 1 .

TABLE I. DESIGN SPECIFICATIONS FOR SYNTHESIS OF MICRORoBOtiC APPENDAGE STRUCTURES

\begin{tabular}{|c|c|}
\hline \multicolumn{2}{|r|}{ Design parameters } \\
\hline parameter & case 1 \\
\hline design domain & $2000 \mu \mathrm{m} \times 2000 \mu \mathrm{m}$ \\
\hline grid/mesh size & $3 \times 3$ \\
\hline $\begin{array}{l}\text { degree of nodal } \\
\text { connectivity }\end{array}$ & 2 \\
\hline end-effector & node: 3 \\
\hline supports & nodes: 1,4 and 7 \\
\hline $\begin{array}{l}\text { number of } \\
\text { parylene-C } \\
\text { elements }\end{array}$ & $\mathrm{i}=28$ \\
\hline $\begin{array}{l}\text { number of PZT } \\
\text { elements }\end{array}$ & $\mathrm{j}=2$ \\
\hline $\begin{array}{l}\text { number of } \mathrm{Si} \\
\text { elements }\end{array}$ & $\mathrm{k}=2$ \\
\hline $\begin{array}{l}\text { value of input } \\
\text { PZT drive voltage }\end{array}$ & $15 \mathrm{~V}$ \\
\hline element modulus & $\begin{array}{c}\text { parylene-C E Eary }=1.3 \mathrm{GPa} \\
\mathrm{Si} \mathrm{E}_{\mathrm{Si}}=170 \mathrm{GPa} \\
\text { PZT E }_{\text {base }}=80 \mathrm{GPa}, \mathrm{E}_{\mathrm{PZT}}=56 \mathrm{GPa}, \mathrm{E}_{\text {top }}=60 \mathrm{GPa}\end{array}$ \\
\hline $\begin{array}{l}\text { element out-of } \\
\text { plane thickness }\end{array}$ & $\begin{array}{c}\mathrm{t}_{\text {pary-T}}=70 \mu \mathrm{m} ; \mathrm{t}_{\text {pary }-\mathrm{L}}=5 \mu \mathrm{m} ; \\
\mathrm{t}_{\mathrm{Si}}=30 \mu \mathrm{m} ; \\
\mathrm{t}_{\text {base }}=0.5 \mu \mathrm{m}, \mathrm{t}_{\mathrm{PZT}}=1 \mu \mathrm{m} ; \mathrm{t}_{\text {top }}=1 \mu \mathrm{m}\end{array}$ \\
\hline $\begin{array}{l}\text { possible values of } \\
\text { element widths }\end{array}$ & $\begin{array}{c}\text { Wpary-T }=50,100,150 \mu \mathrm{m} ; \text { W }_{\text {pary-L }}=20,60,100 \mu \mathrm{m} \\
\mathrm{WSi}=10,40 \mu \mathrm{m} \\
\mathrm{WPZT}=50,100,150 \mu \mathrm{m}\end{array}$ \\
\hline $\begin{array}{l}\text { size of node } \\
\text { wandering space }\end{array}$ & $100 \mu \mathrm{m} \times 100 \mu \mathrm{m}$ \\
\hline $\begin{array}{l}\text { size of node } \\
\text { wandering space } \\
\text { in additional } \\
\text { optimization }\end{array}$ & $100 \mu \mathrm{m} \times 100 \mu \mathrm{m}$ \\
\hline external loads & $\mathrm{F}_{\mathrm{y}}=1 \mu \mathrm{N}, \mathrm{F}_{\mathrm{z}}=1 \mu \mathrm{N}$ \\
\hline PZT elements & $\begin{array}{l}\text { PZT1 }=1-28 \text { (possible values) } \\
\text { PZT2 }=1-28 \text { (possible values) }\end{array}$ \\
\hline Si elements & $\begin{array}{l}\text { Si } 1=1-28 \text { (possible values }) \\
\text { Si } 2=1-28 \text { (possible values })\end{array}$ \\
\hline $\begin{array}{l}\text { parylene-C } \\
\text { elements }\end{array}$ & $\begin{array}{l}\text { parylene } T \text { type }=1-28 \text { (possible values) } \\
\text { parylene } L \text { type }=1-28 \text { (possible values) }\end{array}$ \\
\hline
\end{tabular}

\section{Parameterization}

In the second step of the synthesis process parameterization is done (Fig. 2b), representing the problem and design domain by a set of variables that can be optimized. As the structures are comprised of individual active and passive elements, the ground structure approach (GSA) [14-18] is adopted for the parameterization. The design domain is divided by a number of nodes and beam elements connecting these nodes (Fig. 2b). This serves as an initial solution of the micro-robot appendage design problem, among which optimal solution is searched. Initially all the beam elements in the given structure represent parylene-C elements (Fig. 2c), where during optimization individual parylene elements are replaced with active-PZT and passiveSi elements (Fig. 2d, 2e). The design variables are:
- variables that turn off/on parylene-C elements from the initial ground structure $-\mathrm{v}_{\text {pary }}^{\mathrm{i}}$; permissible values of 1 or $0\left(\mathrm{v}_{\text {pary }}^{\mathrm{i}}=1\right.$ or 0$)$; where 0 means that the selected parylene- $\mathrm{C}$ element is eliminated from the ground structure and 1 that the element is kept. The number of variables (i) is equal to the total number of parylene elements in the structure $\left(\mathrm{n}_{\text {pary }}\right)$;

- variables that select parylene-C elements that will be transformed to a PZT elements $\left(\mathrm{v}_{\mathrm{PZT}}^{\mathrm{j}}\right)$; each variable can have a value between 1 and the total number of parylene-C elements $\left(\mathrm{v}_{\mathrm{PZT}}^{\mathrm{j}}=1\right.$ to $\left.\mathrm{n}_{\text {pary }}\right)$ - here every parylene-C element has a chance to be selected as PZT element. The number of the variable $(\mathrm{j})$ is equal to the number of PZT elements that to be allowed, typically constrained by power budget;

- variables that select parylene-C elements that will be transformed to $\mathrm{Si}$ element $\left(\mathrm{v}_{\mathrm{Si}}^{\mathrm{k}}\right)$; each variable can have a value between 1 and the total number of parylene elements $\left(\mathrm{v}_{\mathrm{Si}}^{\mathrm{k}}=1\right.$ to $\left.\mathrm{n}_{\text {pary }}\right)$ - here every parylene-C element has a chance to become $\mathrm{Si}$ element. The number of variables $(\mathrm{k})$ is equal to the number of Si elements that are permitted, potentially constrained by mass budget.

- variables that selects between $\mathrm{T}$ or $\mathrm{L}$ type of parylene-C elements - $\mathrm{v}^{\mathrm{i}}$ TLpary. Variable can take values 0 or $1\left(v^{\mathrm{i}}\right.$ TLpary $=0$ or 1$)$, where 0 leads to selection of $\mathrm{T}$ and 1 to selection of $\mathrm{L}$ type elements. Every parylene-C element in the structure has a chance to be selected as $\mathrm{T} / \mathrm{L}$ element;

- the width of parylene-C, $\mathrm{Si}$, and PZT elements are also optimized. Thus, for every type of element (parylene L, parylene $\mathrm{T}, \mathrm{Si}$, and $\mathrm{PZT}$ ) variable that defines desired range of element width is introduced ( $w_{\text {paryL }}^{\mathrm{i}}, \mathrm{w}_{\text {paryT, }}^{\mathrm{i}}, \mathrm{w}_{\mathrm{Si}}^{\mathrm{j}}$ and $\mathrm{w}^{\mathrm{k}}$ PZT $)$; variables have discrete values.

- additionally, length (and position) of passive and active elements is optimized, with a node wandering approach. During the optimization, nodes are allowed to "wander" in the ground structure (Fig. $2 b)$. For every node in the structure two variables exist $\left(v^{n}{ }_{n i}, v^{n}{ }_{n j}\right)$, to define the possible range of node wandering in the $\mathrm{x}-\mathrm{y}$ plane (the plane of microstructure fabrication on a silicon wafer).

Topology optimization of micro-robot appendages represents a spatial problem (due to out-off-plane movement), thus passive elements are represented as beam elements with 6 degrees of freedom at each end-node. For active PZT elements, the linear regime of the model presented in [22] is used.

In the ground structure initially all nodes are interconnected (Fig. 2b) i.e. every node is connected with all other nodes via a beam element. Such fully connected structure often leads to solutions of structures that contain overlapping passive/active elements that are difficult to manufacture. Thus, a filter (in form of a computer search algorithm) is developed to eliminate these overlapping elements, prior to the optimization. 


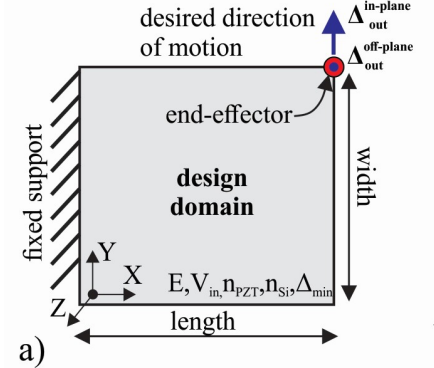

b)

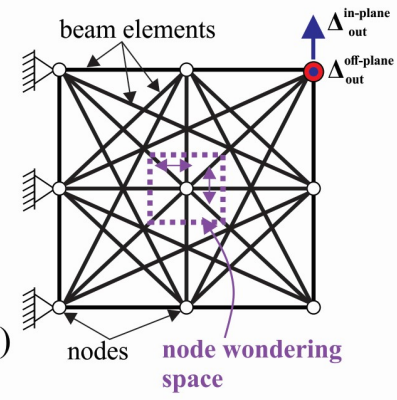

c)
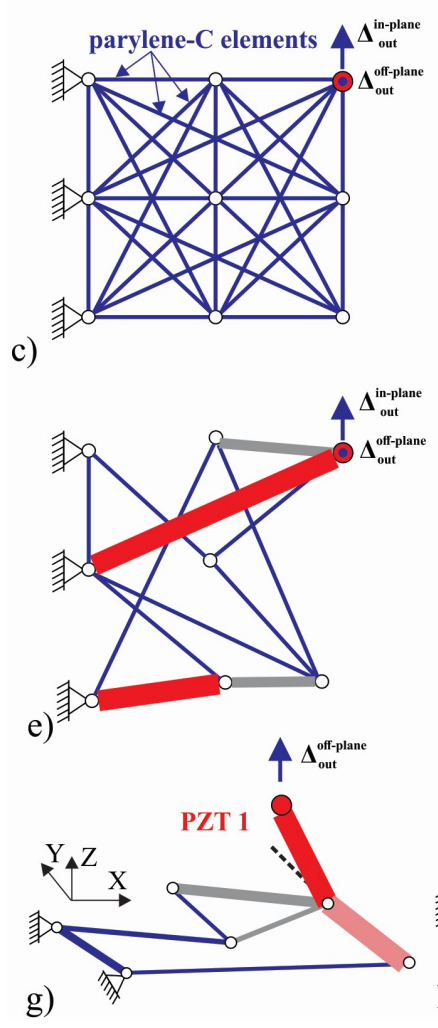

d)
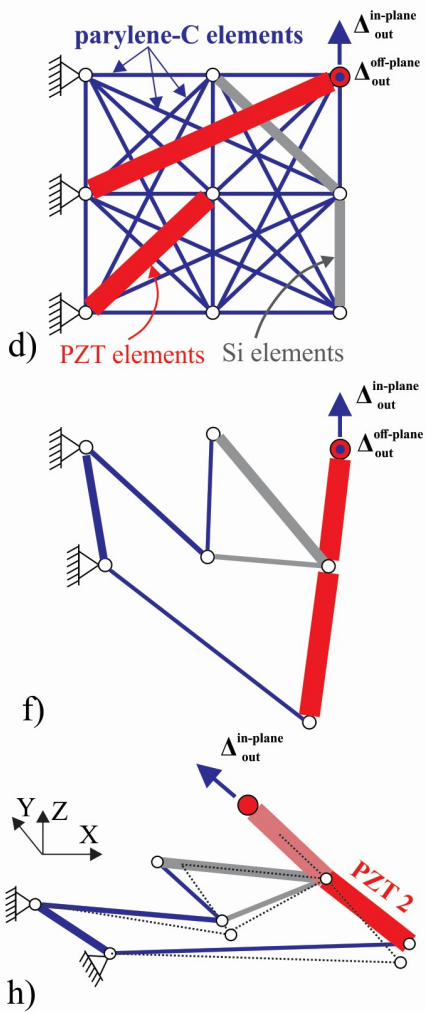

Figure 2. Illustration of the micro-robotic appendage synthesis process for a case of providing motion in in-plane and out-off-plane directions under different PZT activation states: a) definition of problem specifications; b) discretization of the design domain (parameterization); c) initial ground structure with only parylene-C elements; d) ground stucutre with parylene-C, PZT and Si element; e) optimization process (selection between parylene-C, PZT and Si elements); f) example of optimal solution; g) control of end-effector in in-plane direction when PZT 1 is active; h) control of endeffector in out-off-plane when PZT 2 is active.

Obtained partially connected structure is still complex and can lead to solutions that contain structural passive/active elements that cross between nodes of mechanical intersections. Such structures are very difficult to manufacture, since some elements preferred by the optimizer are overlaying and intersecting one another while being intended to move independently. This is usually avoided by discretizing the design domain with a simplified-reduced ground structure (one node is connected only to neighboring nodes) $[12,14,15]$. However, the reduced ground structure cannot always effectively represent the design domain; many good, possible solutions of the given problem may be lost at the beginning. A partially connected ground structure can more effectively represent the design domain, but still, solutions with intersecting elements are obtained [13, 19]. To keep the desired complexity of the ground structure used, and obtain solution without intersections, search filter is developed. This filter in a form of a computer algorithm, searches the intersections between the elements during optimization, where the total number of intersections is minimized.

The design parameters that are used for the discretization of the design domain are given in Table 1.

\section{Optimization}

In the third step of synthesis the optimization is done. The optimization is applied as a search method to find the optimal solution of micro-robot appendage in the given space of design variables (Fig. 2d, 2e). The primary goal of optimization is to realize micro-robot appendage structure that can produce a large difference between end-effector position when different combination of PZT elements are active, while maximizing the end-effector displacement and satisfying the given constraints. In other words, the goal is to maximize the controllability of the end-effector (in different directions) when different combinations of active PZT elements are used.

1) Controllability: The term controllability is borrowed from the control theory, but here controllability is defined as the measure of how well given set of PZT actuators can realize different output states of the micro-robot end-effector. Controllability can be also looked at as a measure of how well the motions of the end-effector, in different directions, are decoupled (realized when different PZT are active). An ultimate goal might be that each PZT actuator in the microrobot structure, when activated, realizes motion/displacement of end-effector solely in one direction. For example, if we have 2 PZT elements in the structure, then the maximal controllability would be achieved as: when only PZT actuator 1 is active, the end-effector realizes solely displacement in the in-plane direction, and when only PZT actuator 2 is active, the end-effector realizes solely displacement in the out-off-plane direction. Controllability is usually represented as a number ranging from 0 to 1 , and is calculated by using the following equation:

$$
\eta_{C}=\left|\frac{\operatorname{det}\left[\vec{d}_{a c t 1} \vec{d}_{a c t 2} \ldots \vec{d}_{a c t N}\right]}{\left|\vec{d}_{a c t 1}\right|\left|\vec{d}_{a c t 2}\right| \ldots\left|\vec{d}_{a c t N}\right|}\right| .
$$

where

$$
\left[\vec{d}_{a c t 1} \vec{d}_{a c t 2} \ldots \vec{d}_{a c t N}\right]=\left[\begin{array}{cccc}
d_{\text {Xact } 1} & d_{\text {Yact } 1} & \ldots & d_{\text {Mact } 1} \\
d_{\text {Xact } 2} & d_{\text {Yact } 2} & \ldots & d_{\text {Mact } 2} \\
\vdots & & & \\
d_{\text {XactN }} & d_{\text {YactN }} & \ldots & d_{\text {Mact } N}
\end{array}\right]
$$

where $\vec{d}_{\text {actN }}$ represents a vector of end-effector displacement (in desired directions of control), when the $N$-th PZT actuator is active. To calculate the displacement vectors, linear FEA is used, implemented in the synthesis algorithm. For this paper, the goal is to realize micro-robot appendages that can control 
end-effector motion in two directions (in-plane and out-offplane), thus two PZT actuators are used. Here each PZT should realize control of end-effector motion in one direction, thus $\mathrm{N}=2$. Controllability represents one of the objectives that need to be maximized during the optimization process.

2) Objective function: The objective function is needed to guide the optimization towards some set of desired goals.

Different formulations of objective functions were explored to find ones suitable for optimizing micro-robot appendages realizing controllable end-effector motion in different directions. The resulting objective function is given in a form:

$$
\max \left[\eta_{C} \cdot d_{E F}^{a c t \min }-\frac{w_{1}}{d_{E F}^{a c t \min }}-w_{2} \cdot d_{e x t}-w_{3} \cdot n_{\mathrm{int}}\right]
$$

where:

$\eta_{C^{-}}$controllability of the micro-robot end-effector; to be maximized and calculated by using (1);

$d_{E F}^{a c t \text { min }}$ - the lowest-displacement of the end-effector among the desired directions of control; maximized in order to realize large displacement in both in-plane and out-off-plane direction; $d_{\text {ext }}$ - end-effector displacement due to external loads; minimized as the micro-robot design should be stiff enough to withstand external loads;

$n_{\text {int }}$ - the total number of intersections in the structure (between passive and active elements) where the goal is to minimize this number so that solutions without intersections are obtained (solutions that can be easily manufactured).

In (3), $w_{1}$ to $w_{3}$ represent weighting constants. Synthesis of micro-robot appendage structures represents a multiobjective optimization problem, reduced to a single objective using these weighting constants. All terms in the (4) are calculated using linear FEA implemented in the synthesis algorithm.

3) Optimization method: Large number of optimization variables (large space of possible solutions) and discrete nature of the given problem (design domain is partially parameterized with discrete elements) motivate us to use discrete evolutionary algorithms. Here, genetic algorithms [23] are applied due to their many advantages (notably, ease of finding global optima over a large space of design variables) and common use in topology optimization $[12,13$, 18]. The genetic algorithm parameters used in the optimal synthesis of the appendage structure are: initial population of 200 designs, a total of 1000 generations, selection function type roulette, crossover probability of $95 \%$, elite count of 2 members, and mutation probability of $9 \%$. The optimization process is done by simultaneously turning off/on parylene-C elements from the initial ground structure, replacing individual parylene-C elements with PZT and Si elements, selecting the type of parylene-C elements $\mathrm{L}$ or $\mathrm{T}$, selecting the width of all the passive/active elements, moving the nodes in different directions/positions, and eliminating the intersection between all the elements (Fig. 2d, 2e). The process is repeated until the solution of the micro-robot appendage structure (optimal topology of the micro-robot appendage) that can best satisfy (3) with a given set of weightings is found (Fig. $2 f-2 h$ ).

\section{RESULTS}

Multiple genetic algorithm optimizations are run for different sizes of node wandering space in both initial and additional optimization (Table 1), and with different values of weighting constants $w$. It is important to mention that after the optimal solution of a micro-robot appending structure is obtained, additional node wandering optimization is done with the goal to achieve larger output displacements of the end-effector while keeping the same values of controllability. For the final structures, only nodes are allowed to wander in larger wandering space while fixing other parameters (thickness, widths, selection of element type, topology). This is done in order to explore a broader range of possible optimal solutions, while keeping a similar topology.

Multiple resulting structures have been obtained. Fig. 3 shows some representative results of micro-robot appendage topology optimization using PZT, polymer and silicon elements. In general, optimized structures have a similar trade-off between stiffness against external loads and rangeof-motion in the vertical direction $(\delta z)$ as a single piezoelectric cantilever of $150 \mu \mathrm{m}$ width with a similar range of total piezoelectric area. This is effectively limited by the total force or work available from the piezoelectric elements to drive motion, though there is some ability to outperform the baseline by manipulating location of the load. Importantly, however, comparable motion in the in-plane direction can also be realized by optimized designs $(\delta y)$, with controllability between directions of motion that is unique to these designs. Fig. 4 shows the same resulting micro-robot appendage structures that realize motion control of endeffector in in-plane and out-off-plane direction, when different PZT are active. In most cases high value of controllability is realized (above 99\%). This means that the obtained structures realize nearly pure end-effector motion in desired direction, when the corresponding PZT is active.

\section{DISCUSSION AND CONCLUSIONS}

Despite limitations, topology optimization may provide advantages for micro-robot development based on thin-film piezoelectric actuators, which to date has mostly been reliant on designer experience and done through prototype and iteration processes. As robot fabrication by MEMS processes can be time consuming and expensive, modeling and optimization prior to construction can be highly advantageous. The optimization method presented in this paper may lead to one general tool for optimal design of micro-robots.

\section{ACKNOWLEDGMENT}

We thank Dr. Jongsoo Choi and Dr. Minchul Shin for contributions to models of individual model elements and information on fabrication constraints. Research was partially supported by Academy of Finland Research Council for Natural Sciences and Engineering (No. 318390). 


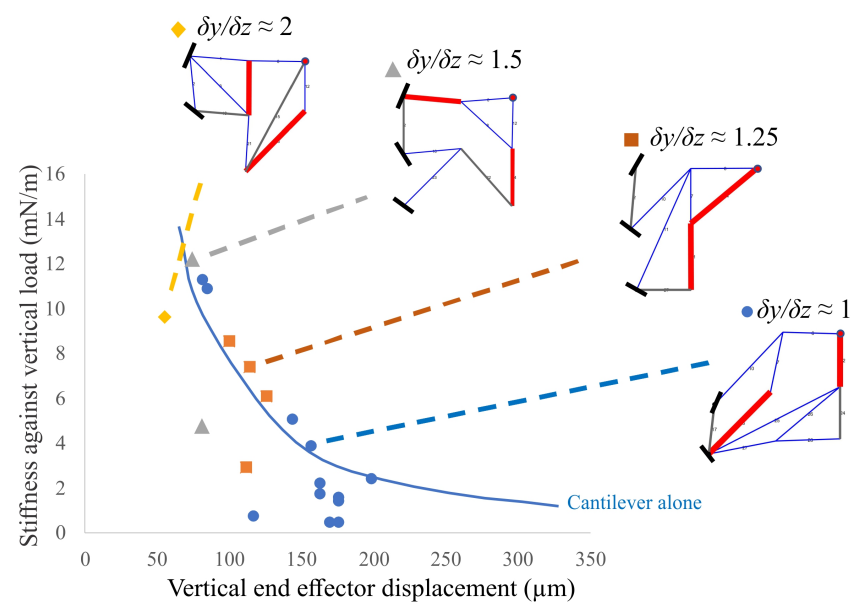

Figure 3. Representative results of topology optimization using PZT, polymer, and silicon link elements. Overall load capacity vs. range of motion remains similar to that of a standalone PZT cantilever, but structures add controllable motion in multiple axes, in these cases permitting equal or larger deformation in lateral $(\delta y)$ versus vertical $(\delta z)$ directions.
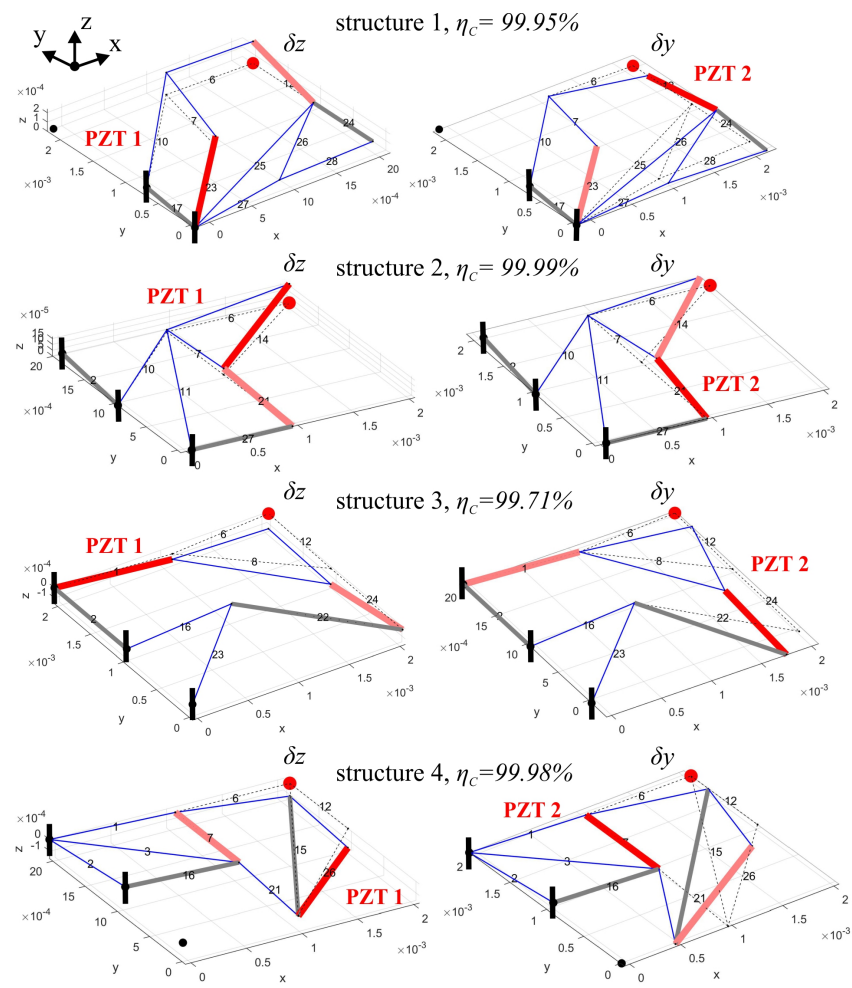

Figure 4. Control of the end-effector motion in out-of-plane (images - left) and in-plane (images - right) directions for select optimized micro-robotic appendage structures, when different PZT are active. High value of controllability is achieved (here shown in \%).

\section{REFERENCES}

[1] K. Oldham, J. Pulskamp, R. Polcawich, P. Ranade, and M. Dubey, "Thinfilm piezoelectric actuators for bio-inspired micro-robotic applications," Integrated Ferroelectrics, vol. 95, no. 1, pp. 54-65, Jun 2007.

[2] J. E. Huber, N. A. Fleck, and M. F. Ashby, "The selection of mechanical actuators based on performance indices," in Proc. Mathematical, Physical and Engineering Sciences, vol. 453, no. 1965, pp. 2185-2205, Oct 1997.

[3] B. Edamana and K. R. Oldham, "Optimal low-power piezoelectric actuator control with charge recovery for a microrobotics leg," IEEE/ASME Transactions on Mechatronics, vol. 18, no. 1, pp. 251262, Feb 2013.
[4] M. Karpelson, G.-Y. Wei, and R. J. Wood, "A review of actuation and power electronics options for flapping-wing robotic insects," International Conference on Robotics and Automation, Pasadena, CA, May 2008.

[5] K. Sugita, D. Tanaka, S. Ono, S. Chiba, K. Iwata, Y. Han, et al., "SMA actuator and pulse-type hardware neural networks IC for fast walking motion of insect-type MEMS microrobot," in Advanced Intelligent Mechatronics (AIM), 2016 IEEE International Conference on, 2016, pp. 431-435.

[6] R. Murthy, A. N. Das, D. O. Popa, and H. E. Stephanou, "ARRIpede: An assembled die-scale microcrawler," Advanced Robotics, vol. 25, pp. 965-990, 2011.

[7] J. Choi, M. Shin, R. Q. Rudy, C. Kao, J. S. Pulskamp, R. G. Polcawich, and K. R. Oldham, "Thin-film piezoelectric and highaspect ratio polymer leg mechanisms for millimeter-scale robotics," International Journal of Intelligent Robotics and Applications, vol. 1, no. 2, pp. 180-194, Jun 2017.

[8] J. Qu, J. Choi, and K. R. Oldham, "Dynamics structural and contact modeling for a silicon hexapod microrobot," Journal of Mechanism and Robotics, vol. 9, no. 6, pp. 061006, Sep 2017.

[9] L .L. Howell, S. P. Magleby, and B. M. Olsen, Handbook of Compliant Mechanisms, Chichester: John Wiley \& Sons, 2013.

[10] S. Chu, L. Gao, M. Xiao, Z. Luo, and H. Li, "Stress-based multi-material topology optimization of compliant mechanisms," Internacional Journal of Numerical Methods in Engineering, vol. 113, pp. 1021-1044, February 2018.

[11] L. Sebastian, P. Gräser, T. Räder, S. Henning, R. Theska, and L. Zentner, "Influence of geometric scaling on the elasto-kinematic properties of flexure hinges and compliant mechanisms," Mechanism and Machine Theory, vol. 125, pp. 220 - 239, July 2018.

[12] R. Parsons and S. L. Canfield, "Developing genetic programming techniques for the design of compliant mechanisms," Structural and Multidisciplinary Optimization, vol. 24, pp. 78-86, August 2002.

[13] A. Milojević and N. D. Pavlović, "Software for the synthesis of compliant mechanisms without intersecting elements," Facta Universitatis-Series: Mechanical Engineering, vol. 11, pp. 153-168, December 2013.

[14] A. Saxena and G. K. Ananthasuresh, "On an optimal property of compliant topologies," Structural and Multidisciplinary Optimization, vol. 19, pp. 36-49, March 2000.

[15] A. Saxena, "Synthesis of compliant mechanisms for path generation using genetic algorithm," Journal of Mechanical Design, vol. 127, pp. 745-752, Julay 2005

[16] R. A. Narayana, M. Nandan, S .D. Kumar, and G. K. Ananthasuresh, "Miniature Compliant Grippers With Vision-Based Force Sensing," IEEE Transactions on Robotics, vol. 26, pp. 867-877, October 2010.

[17] A. Saxena, "Topology design of large displacement compliant mechanisms with multiple materials and multiple output ports," Structural and Multidisciplinary Optimization, vol. 30, pp. 477-490, December 2005.

[18] A. Milojević and N. D. Pavlović, "Development of a new adaptive shape morphing compliant structure with embedded actuators," Journal of Intelligent Material Systems and Structures, vol. 27, pp. 1306-1328, Jun 2016.

[19] J. Prasad and A .R. Diaz, "Synthesis of bistable periodic structures using topology optimization and a genetic algorithm," Journal of Mechanical Design, vol. 128, pp. 1298-1306, November 2006.

[20] R. E. K. Moussa, M. Grossard, M. Boukallel, N. Chaillet, and A. Hubert, "Observation-oriented design of a monolithic piezoelectric microactuator with optimally integrated sensor," 41st International Symposium on Robotics and ROTOTIK 2010., Jun 2010, Munich, Germany. Verlag, 1, pp.954-961, 2010.

[21] D. Ruiz and O. Sigmund, "Optimal design of robust piezoelectric microgrippers undergoing large displacements," Structural and Multidisciplinary Optimization, vol. 57, pp. 71-82, January 2018.

[22] K. R. Oldham, J. S. Pulskamp, R. G. Polcawich, and M. Dubey, "Thinfilm PZT lateral actuators with extended stroke," Journal of Microelectromechanical Systems, vol. 17, no. 4, pp. 890-899, Aug 2008.

[23] R. L. Haupt and S .E. Haupt, Practical Genetic Algorithms. New Jersey: John Wiley \& Sons, 2004. 